\title{
Does vitamin D supplementation impact plasma cathelicidin, human beta defensin 2 and intestinal permeability in stable Crohn's disease? - Results from a randomised, double blind placebo controlled study
}

\author{
T. Raftery ${ }^{1}$, A. Martineau ${ }^{2}$, C. Greiller ${ }^{2}$, C. S. Lee ${ }^{3}$, D. McNamara ${ }^{3}$, S. Ghosh $^{4}$, J. Meddings $^{4}$ \\ and M. O'sullivan ${ }^{1}$ \\ ${ }^{1}$ Dept. of Medicine, Trinity Centre for Health Science, St. James's Hospital, Dublin 8, Ireland, ${ }^{2}$ Blizard Institute, Barts \\ and The London School of Medicine and Dentistry, Queen Mary University of London, London E1 2AB, ${ }^{3}$ Dept. of Medicine, \\ Trinity Centre for Health Science, Tallaght Hospital, Dublin 24, Ireland, Dept. of Medicine and ${ }^{4}$ Dept. of Medicine, \\ University of Calgary, Canada
}

There is emerging evidence that vitamin D (vitD) supplementation may prevent relapse (1) and have therapeutic potential in Crohn's disease (CD)(2), however, the underlying mechanisms remain unclear. We propose that effects on intestinal permeability (IP) and on the antimicrobial peptides cathelicidin (LL37) and human beta defensin2 (HBD2) may be important. The aim of the study was to investigate the effect of vitD supplementation on IP, and on the plasma concentrations of LL37 and HBD2 as potential mechanisms in maintaining remission in $\mathrm{CD}$.

In a double-blind placebo-controlled study, $27 \mathrm{CD}$ patients in remission were randomly assigned to $2000 \mathrm{IU}$ oral vitD3/d or placebo for $3 \mathrm{mo}$ as adjunctive therapy during winter. Permeability was assessed at 0 and $3 \mathrm{mo}$ by the quadruple sugar test. Small bowel IP was expressed as lactulose:mannitol recovery (LMR), gastroduodenal and colonic permeability as sucrose and sucralose excretion (mg). Plasma LL37 (ng/ml) and HBD2 (pg/ml) were determined by commercial ELISA at baseline and 3mo. Disease activity [Crohn's disease activity index (CDAI)], CRP (mg/l), and serum 25-hydroxy vitD (25OHD) (nmol/l) were measured at 0 and 3mo. Ethical approval and informed written consent were obtained and the research was performed in accordance to the Declaration of Helsinki.

The mean (sd) age of subjects was 36.63 (11.73)y. At enrolment, mean (sem) CRP [2.39 (0.53)] and CDAI [92.35 (13.23)] were consistent with disease remission. At 3mo mean (95\% CI) 25OHD levels were significantly higher in the vitD group 91.6 (75.5-107.6) $\mathrm{nmol} / \mathrm{l}$ than in the placebo group $40.4(30.4-50.4) \mathrm{nmol} / \mathrm{l}(p<0.001)$. Baseline LMRs were normal $(<0.025)$ in $72 \%$ of the vitD group and in all controls. At $3 \mathrm{mo}$, there was a significant increase in LMRs $(p=0.010)$ (fig. 1) and sucrose excretion $(p=0.030)($ fig.2) in the controls but these parameters were unchanged in the vitD group $(p=0.899$ and $p=0.590)$.

At 3mo the vitD group had lower mean (sem) concentrations of LL37 [181.9 (59.3) v. 193.9 (64.3), $p=0.124]$ and HBD2 [2293.2 (472.5) $\mathrm{pg} / \mathrm{ml}$ v. 2687.9 (570.2) $\mathrm{pg} / \mathrm{ml}, p=0.064]$ compared to the control group. Subjects with $25 \mathrm{OHD}$ levels $>=100 \mathrm{nmol}$ had significantly lowerLL37 concentrations [154.7 (40.9) ng/ml] compared to those with levels $<100 \mathrm{nmol} / 1$ [(333.8 $(158.7) \mathrm{ng} / \mathrm{ml}, p=0.046]$.

At $3 \mathrm{mo}$ there was no change in CDAI. However, in the vitD-treated group, 25OHD levels were significantly inversely associated with $\mathrm{CRP}$ (rho $=-0.80, p=0.002$ ) and severity of diarrhoea (no. of liquid/very soft stools/week) (rho $=-0.58 ; p=0.046$ ) at $3 \mathrm{mo}$.

In conclusion this study, to our knowledge, is the first to demonstrate that treatment with $2000 \mathrm{IU}$ vitD3/d in CD was accompanied by an apparent maintenance of IP and a decrease in LL37 and HBD2 concentrations in CD. These findings may provide evidence of novel mechanisms underlying the potential therapeutic effects of adjuvant vitD therapy in $\mathrm{CD}$ and merit further study.

1. Jørgensen SP, Agnholt J, Glerup H (2010) 32(3), 377-83.

2. Raftery T, O'Morain C, O'Sullivan M (2012) Current Drug Metab 13(9), 1294-302. 